
\title{
pneumonia
}

Case Report

\section{Salmonella empyema: a case report}

\section{Shivanshan Pathmanathan ${ }^{a}$, Suminda Welagedara ${ }^{a, c}$, Petra Dorrington ${ }^{b, c}$, Siddharth Sharma ${ }^{a, c}$}

a'Department of Medicine, Gold Coast University Hospital, Southport, Queensland, Australia; 'Department of Microbiology, Gold Coast University Hospital, Southport, Queensland, Australia; 'School of Medicine, Griffith University, Southport, Queensland, Australia

Corresponding author: Dr Shivanshan Pathmanathan, Department of Medicine, Gold Coast University Hospital, 1 Hospital Boulevard, Southport, Queensland 4215, Australia. Phone: +61 430289575. Email: shivvyp89@gmail.com

Author contributions: All the authors met ICMJE authorship criteria. SP, SW, PD, SS conceived and designed the research plan. SP, SW, PD, SS collected and conducted the data analysis and interpretation. SP wrote the first draft of the manuscript. SW, PD, SS critically reviewed the manuscript for important intellectual content. All authors agree with the results and conclusions. All authors approved the final version of the manuscript.

Received 23 June 2015; Accepted 4 November 2015; Published 17 November 2015

Citation: Pathmanathan S, Welagedara S, Dorrington P, Sharma S. Salmonella empyema: a case report. pneumonia 2015;6:120-124

\section{Abstract}

Non-typhi Salmonella enterica infection rarely presents as a pleural empyema, with only 31 cases published in the literature over the last century. We report a case of an 85-year-old female with worsening shortness of breath and pleuritic chest pain, and a chest radiograph showing a right-sided pleural effusion. Thoracocentesis revealed Salmonella enterica serovar Typhimurium to be the causative organism. This was on a background of recurrent pleural effusion secondary to congestive heart failure, with thoracocentesis one month previously showing a transudative picture. This case highlights the possibility of $S$. enterica as a differential diagnosis in the management of pleural effusions.

Keywords: Salmonella, enterica, empyema, pleural, pulmonary 


\section{Introduction}

Salmonella spp., first described in the 1880s by Salmon and Smith [1,2], are Gram-negative, non-sporeforming, facultative anaerobic bacilli of the family Enterobacteriaeceae. Salmonella enterica serovars Typhi and Paratyphi produce a spectrum of clinical presentations including enteric fever. Non-typhi $S$. enterica serovars most commonly present with gastroenteritis [3]. Extra-intestinal manifestations include bacteraemia and focal infections ranging from endovascular arteritis, endocarditis, septic arthritis, osteomyelitis, urinary tract infection to splenic abscess. Non-typhi $S$. enterica serovars rarely cause pleuropulmonary disease, in particular pleural empyema. Only 1 case of non-typhi $S$. enterica empyema has been reported in Australia [4]. This case report presents a case of unilateral empyema caused by Salmonella enterica serovar Typhimurium, and a review of the literature.

\section{Case report}

An 85-year-old female of English heritage presented with a 3-day history of increasing right-sided pleuritic chest pain associated with increased shortness of breath and increased non-productive cough. There was no fever or increased sputum production. The patient did not report diarrhoea, abdominal or flank pain on admission, or in the 12 months leading to admission. Significant past medical history included left ventricular heart failure complicated by previous presentations with right-sided pleural effusions requiring thoracocentesis. Thoracocentesis 1 month before this admission had shown a transudative picture with no bacterial growth on culture. Other clinical history included ischaemic heart disease, severe mitral valve stenosis, atrial fibrillation, hypertension, mild neutropenia $\left(1.25 \times 10^{9} / \mathrm{I}\right)$, pending bone marrow aspirate, and Streptococcus gallolyticus subsp. gallolyticus infective endocarditis in 2010. There was no suggestion of prior $S$. enterica infection, cholelithiasis or urolithiasis. There was no history of travel outside Australia in the preceding 12 months.

Physical examination revealed an oxygen requirement (4 litres to maintain saturations greater than 95\%), respiratory rate of 16 breaths/min, tachycardia (100-120 beats/min, irregular), normal blood pressure (120/70 $\mathrm{mmHg}$ ) and afebrile. Cardiovascular examination revealed a jugular venous pressure elevated at $7 \mathrm{~cm}$ and a Grade 3 pansystolic murmur over the mitral region, radiating to the axilla. Respiratory examination revealed dullness to percussion in the right middle to lower zones, with reduced breath sounds noted in the same area. The abdomen was soft on palpation and no tenderness was elicited. There was bilateral pitting oedema to the level of the knees. Initial pathology revealed a normal white blood cell count of $2.7 \times 10^{9} / \mathrm{I}$ with $46 \%\left(1.25 \times 10^{9} / \mathrm{I}\right)$ neutrophils and $30 \%$ $\left(0.82 \times 10^{9}\right) /$ l lymphocytes. C-reactive protein (CRP) was elevated at $268 \mathrm{mg} / \mathrm{l}$.

The chest radiograph revealed opacification of the lower two-thirds of the right hemithorax, with loss of the right costophrenic angle. Underlying collapse was also noted. The chest radiograph can be viewed in Figure 1. A thoracocentesis was subsequently performed. Analysis of pleural fluid revealed a white cell count of $1,350 \times$ $10^{6} / \mathrm{l}$, a protein concentration of $35 \mathrm{~g} / \mathrm{l}$, and a lactate

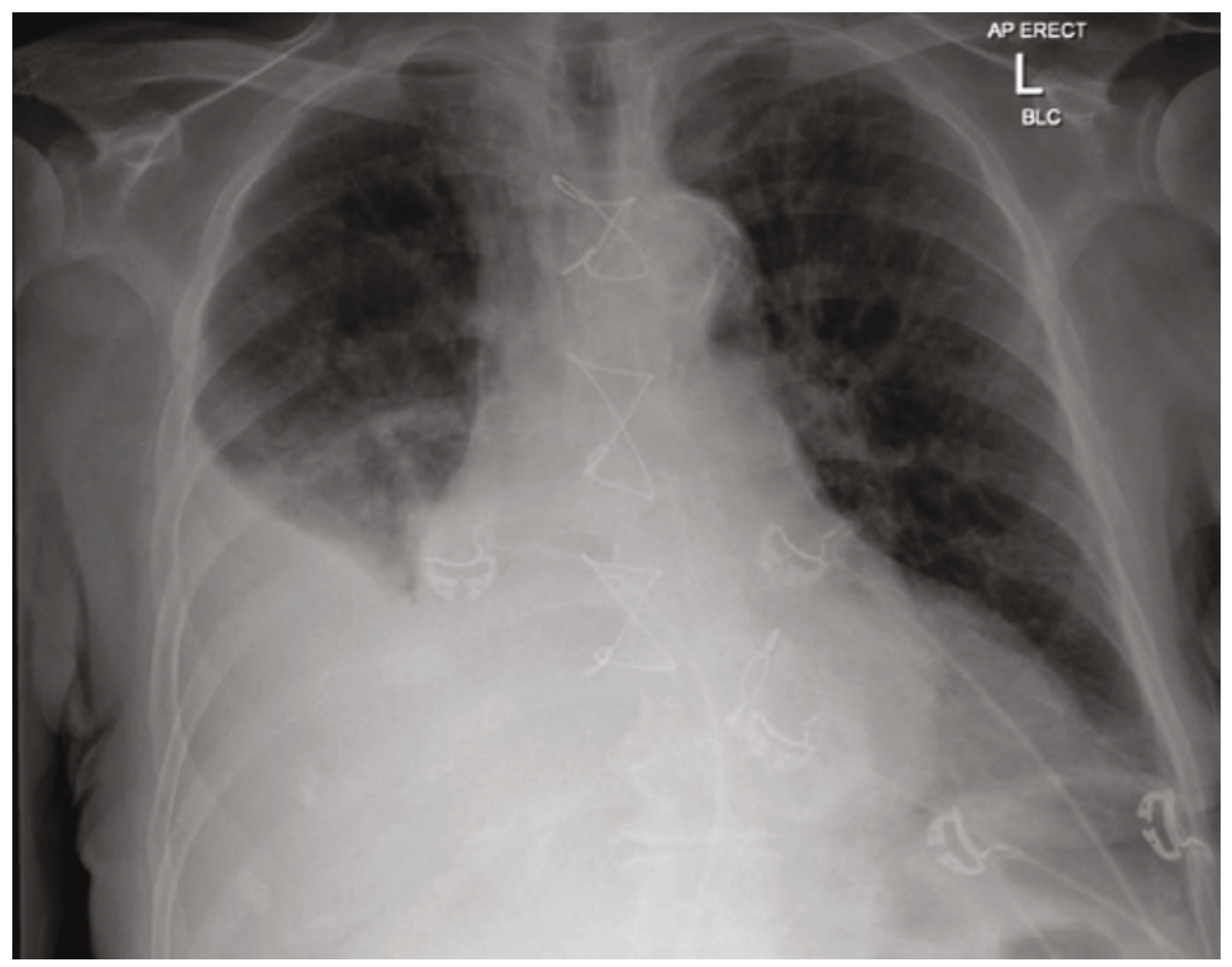

Figure 1 Chest radiograph on presentation showing evidence of right-sided pleural effusion 
dehydrogenase level of $590 \mathrm{U} / \mathrm{l}$. These values were consistent with exudate. The $\mathrm{pH}$ of the sample was 7.1, indicating empyema. Gram-negative bacilli were seen in a Gram-stained film of the fluid. A second thoracocentesis was performed 2 days later, again showing Gram-negative bacilli. Subsequent cultures grew $S$. enterica serovar Typhimurium. Three sets of blood cultures and 2 stool cultures taken prior to starting antibacterial therapy were negative for S. enterica.

The intended management of the patient included insertion of a chest tube (tube thoracostomy), and a 4to 6-week course of ceftriaxone, depending on clinical response. However the patient declined a chest drain, and wished to cease ceftriaxone after two days. The patient received palliative care aiming for symptom control. Challenges included the management of severe right sided pleuritic chest pain, dyspnoea and nausea, requiring continuous subcutaneous infusion of fentanyl, haloperidol and maxalon. Hydromorphone was used for breakthrough analgesia. The patient passed away comfortably in the palliative care unit 5 days after stopping the antibiotics.

\subsection{Ethics statement}

This case report complies with the current policies of Gold Coast Hospital and Health Service, Gold Coast, Queensland, Australia (GCHHS) for a deidentified patient who is deceased. Patient consent for publication was obtained and recorded in the electronic medical records. For the purpose of publication the HREC reference number is: HREC/15/QGC/190.

\section{Discussion}

A literature review published by Crum [5] only identified 28 cases of $S$. enterica empyema up to 2005 . The literature review included adult and paediatric patients and excluded empyema caused by S. enterica serovars Typhi and Paratyphi. From 2005 to 2015, 2 further cases of nontyphi $S$. enterica empyema have been identified in the literature [6] [7]. Only 1 published case has been identified in Australia (1991) [4]. The patient was a 70-year-old male with a background of lymphoma in remission who presented with a 6-week history of chest pain, and was found to have $S$. enterica serovar Typhimurium. The patient was managed with intravenous ampicillin, and decortication. The underlying cause was not determined. S. enterica serovar Typhi also rarely presents with pleural empyema, with only 7 cases being reported in the literature, predominantly in the paediatric population [8] [9].

Although a rare diagnosis, trends in co-morbidities and symptoms have been reported. It is common for patients to be immunosuppressed secondary to co-morbidities such as human immunodeficiency virus infection, diabetes, malignancy, iatrogenic (prednisone, azathioprine, chemotherapy), iron overload, and chronic renal insufficiency [4] [5] [7] [10] [11] [12]. Pre-existing lung disease has also been noted to be present in $38 \%$ of the patients, suggesting a propensity to adhere to damaged lung tissue [5] [13] [14] [15]. The patient reported in this study had a combination of prior lung disease with prior pleural effusion, and immunosuppression secondary to a Grade 1 neutropenia. Although the patient did not have a significant travel history, other risk factors would include travel to endemic areas such as India, South-East Asia and Africa [16].

There was no history of recent or prior Salmonella infection in this patient, although prior reports have shown only $33 \%$ of patients had a history of gastrointestinal illness [5]. A second interesting feature is that she had presented 4 weeks prior with similar symptoms. Thoracocentesis was performed and showed a transudative picture, attributed to congestive heart failure. Therefore, pleural fluid chemistry can change, and results of recent pleural fluids sampling should be used with caution in the context of changed clinical scenario. This is particularly true for patients with risk factors such as heart failure or lung cancer who may present repeatedly with pleural effusions. Nontyphi S. enterica serovars empyema commonly presents with a leukocytosis [5]. The lack of leukocytosis in this case can be attributed to the immunosuppression secondary to neutropenia and possible bone marrow involvement. In this case, CRP was elevated and would have been the more appropriate method to monitor the inflammatory response.

Transmission of non-typhi Salmonella predominantly occurs through infected food products such as eggs and dairy products. Most commonly, patients present with local symptoms of gastroenteritis [3]. The hypothesised pathogenesis of focal infections such as empyema includes bacteraemia with subsequent seeding in patients presenting with positive blood cultures for $S$. enterica [10] [12] [17]. Other causes include seeding from nearby infection such as the spleen and pancreas, with subsequent transdiaphragmatic tracking [7] [18] [19] [20] [21] [22] [23]. Therefore, isolation of an enteric organism, such as Salmonella, from a pleural effusion should prompt clinicians to exclude an intra-abdominal source of infection.

Our observations in this case report are consistent with previous cases, which showed no preceding history of Salmonella, blood cultures were negative and stool cultures were negative [5]. In these cases, only $30 \%$ had positive blood cultures, while $39 \%$ had positive stool cultures. It is suspected that non-typhi Salmonella may be dormant in the reticulo-endothelial system, with subsequent re-activation and haematogenous spread [24]. It should also be noted that blood cultures are regularly negative in Salmonella bacteraemia due to low bacterial load [25]. The sensitivity of detection by blood culture also declines with longer duration of the illness [26].

Prior case reports used an extensive range of antibiotics in 
the treatment of Salmonella empyema [27]. Traditionally, ampicillin, chloramphenicol, and cotrimoxazole were used in the management of non-typhi Salmonella infections. However, in light of increasing resistance, third-generation cephalosporins are commonly empirically used until further susceptibility to quinolones is available. The effective utilisation of antibiotics improves outcomes [24].

In conclusion, Salmonella empyema is an uncommon presentation, with only 31 case reports published in the last century. It can be a difficult diagnosis, with a lack of gastrointestinal symptoms, a lack of raised leucocytes, or a lack of positive stool or blood cultures to assist with diagnosis. Therefore, although rare, Salmonella as a cause of pleural effusion should remain a differential diagnosis.

Funding: The authors have no funding to report.

Competing interests: All authors declare no competing interests.

Provenance and peer review: Not commissioned; externally peer reviewed.

Copyright: This is an open-access article distributed under the terms of the Creative Commons Attribution License, which permits unrestricted use, distribution, and reproduction in any medium, provided the original author and source are credited.

\section{References}

1. Salmon DE, Smith T. The bacterium of swine-plague. Am Month Micr J 1886; 7:204

2. Smith T. The hog-cholera group of bacteria. US Bur Anim Ind Bull 1894;6:640

3. Hohmann EL. Nontyphoidal salmonellosis. Clin Infect Dis 2001;32:263-9.http://dx.doi. org/10.1086/318457 PMID:11170916

4. CistulliPA, BarnesDJ, Young GA.Salmonella empyemain a patient with lymphoma. Aust NZJ Med 1991;21:2467.http://dx.doi.org/10.1111/j.1445-5994.1991. tb00454.x PMID:1872755

5. Crum NF. Non-typhi Salmonella empyema: case report and review of the literature. Scand J Infect Dis 2005;37:852-7.http://dx.doi. org/10.1080/00365540500264944 PMID:16308220

6. Kam JC, Abdul-Jawad S, Modi C Abdeen Y, Asslo F, Doraiswamy V, et al. Pleural Empyema due to Group D Salmonella. Case Rep Gastrointest Med 2012; 2012: 524561. http://dx.doi.org/10.1155/2012/524561 PMID:23056966

7. Woo JW, Tam JK, Chan DS, Ying LS. Uncovering what lies beneath a Salmonella Enterica empyema. BMJ Case Rep 2015. http://dx.doi.org/10.1136/bcr-2015211351 PMID:26336186

8. Afridi $\mathrm{FI}$, Farooqi BJ, Hussain A. Pleural empyema due to Salmonella typhi. J Coll Physicians Surg Pak 2012;22:803-5. PMID:23217492

9. Nandan D, Jhavar L, Dewan V, Bhatt GC, Kaur N. A case of Empyema thoracic due to Salmonella typhi in 18-month-old child: an unusual cause. J Lab Physicians 2012;4:45-7.http://dx.doi.org/10.4103/09742727.98673 PMID:22923923
10. Colebunders $R$, Verstraeten $T$, Van den Abbeele K, Stappaert I, Van Meerbeeck J. Salmonella typhimurium empyema in an AIDS patient. Int J STD AIDS 1995;6:287-8. PMID:7548294

11. Lenhard RE Jr, Densen $P$, Lenhard RE. Salmonella empyema and Hodgkin's disease. South Med J 1975;68:363-6. http://dx.doi. org/10.1097/00007611-197503000-00023 PMID:1091004

12. Bieber E, Quinn JP, Venezio FR, Miller JB. Salmonellal empyema in a heart transplant recipient. J Heart Transplant 1989;8:262-3. PMID:2661777

13. Kuncaitis J, Okutan AM. Empyema due to Salmonella typhimurium. Am Rev Respir Dis 1961;83:741-3. PMID:13755117

14. Burney DP, Fisher RD, Schaffner W. Salmonella empyema: a review. South Med J 1977;70:375-7. http://dx.doi.org/10.1097/00007611-19770300000041 PMID:322305

15. Kate $\mathrm{P}$, Osei $\mathrm{K}$, Chiemchanya $\mathrm{S}$, Zatuchni J. Empyema due to Salmonella typhimurium with underlying alveolar cell carcinoma. South Med J 1984;77:234-6. PMID:6322352

16. Majowicz SE, Musto J, Scallan E, Angulo FJ, Kirk M, O'Brien SJ et al. International Collaboration on Enteric Disease 'Burden of Illness' Studies. The global burden of nontyphoidal Salmonella gastroenteritis. Clin Infect Dis 2010;50:882-9. PMID:20158401

17. Rim MS, Park CM, Ko KH, Lim SC, Park KO. Pleural empyema due to Salmonella: a case report. Korean J Intern Med 2000;15:138-41. PMID:10992728

18. Sethia B, Reece IJ, Davidson KG. Empyema, subphrenic abscess and pyaemic splenic necrosis. A rare complication of Salmonella enteritidis infection. J R Coll Surg Edinb 1985;30:204-5. PMID:3900372

19. Roguin A, Gavish I, Ben Ami H, Edoute Y. Pleural empyema with Salmonella mendoza following splenic abscesses in a patient with myelodysplastic syndrome. Isr Med Assoc J 1999;1:195-6. PMID:10731335

20. Charles TJ, Hariraj R. Salmonella empyema following splenic biopsy. Respir Med 1998;92:968-9. PMID:10070573

21. Prigogine T, Fastrez R, Glupczynski Y. Empyema due to Salmonella dublin. Eur J Respir Dis 1986;68:73-4. PMID:3948937

22. Sethia B, Reece IJ, Davidson KG. Empyema, subphrenic abscess and pyaemic splenic necrosis. A rare complication of Salmonella enteritidis infection. J R Coll Surg Edinb 1985;30:204-5. PMID:3900372

23. Buscaglia AJ. Empyema due to splenic abscess with Salmonella newport. JAMA 1978;240:1990. PMID:691225

24. Aguado JM, Obeso G, Cabanillas JJ, FernándezGuerrero M, Alés J. Pleuropulmonary infections due to nontyphoid strains of Salmonella. Arch Intern Med 1990;150:54-6.http://dx.doi.org/10.1001/ archinte.1990.00390130070008 PMID:2404482

25. Farooqui BJ, Khurshid $M$, Ashfaq MK, Khan MA. Comparative yield of Salmonella typhi from blood and bone marrow cultures in patients with fever of 
unknown origin. J Clin Pathol 1991;44:258-9.http:// dx.doi.org/10.1136/jcp.44.3.258 PMID:2013632

26. Coleman W, Buxton BH. The bacteriology of the blood in typhoid fever: an analysis of 1602 cases. Am J Med Sci 1907;133:896-903.

27. Glynn MK, Bopp C, Dewitt W, Dabney P, Mokhtar
M, Angulo FJ. Emergence of multidrug-resistant Salmonella enterica serotype typhimurium DT104 infections in the United States. N Engl J Med 1998;338:1333-8.http://dx.doi.org/10.1056/ NEJM199805073381901 PMID:9571252 